\title{
Frontières
}

\section{DUNN, Katherine, Un amour de monstres. Paris, F1RST, 1990}

\section{Jean-François Chassay}

Volume 25, numéro 2, 2013

URI : https://id.erudit.org/iderudit/1024945ar

DOI : https://doi.org/10.7202/1024945ar

Aller au sommaire du numéro

Éditeur(s)

Université du Québec à Montréal

ISSN

1916-0976 (numérique)

Découvrir la revue

Citer ce compte rendu

Chassay, J.-F. (2013). Compte rendu de [DUNN, Katherine, Un amour de monstres. Paris, F1RST, 1990]. Frontières, 25(2), 137-143.

https://doi.org/10.7202/1024945ar

Ce document est protégé par la loi sur le droit d'auteur. L'utilisation des services d'Érudit (y compris la reproduction) est assujettie à sa politique d'utilisation que vous pouvez consulter en ligne.

https://apropos.erudit.org/fr/usagers/politique-dutilisation/
Cet article est diffusé et préservé par Érudit.

Érudit est un consortium interuniversitaire sans but lucratif composé de l’Université de Montréal, l'Université Laval et l'Université du Québec à Montréal. Il a pour mission la promotion et la valorisation de la recherche. https://www.erudit.org/fr/ 


\section{DUNN, Katherine}

\section{Un amour de monstres}

Paris, F1RST, 1990

\section{Jean-François Chassay}

Professeur, Département d'études littéraires, Université du Québec à Montréal

L'Apocalypse, si on veut l'entendre dans son sens courant, soit une catastrophe, un désastre, est nécessairement monstrueuse puisqu'elle est hors-norme. Comment définir un monstre? Question difficile : un monstre n'existe qu'à travers le regard de l'autre - c'est le génie de Mary Shelley d'avoir insisté, par de multiples biais métaphoriques, sur la présence du regard porté sur la différence pour mieux la repousser. Si le concept de monstre a évolué au fil des siècles, il reste toujours en dialogue avec la norme et, en ce sens, une affaire de société. Une des qualités du livre de Katherine Dunn, Geek Love (Un amour de monstre, dans sa traduction française), dont il sera question ici, est de proposer différents sens possibles de la figure du monstre en nous conduisant, de manière de plus en plus accélérée, vers une apocalypse qui paraît tôt inévitable. La romancière américaine nous révèle la figure du monstre avec une envergure difficile à dépasser.

La version originale anglaise, intitulée Geek Love joue sur les multiples significations du mot geek. On l'utilise pour désigner un individu obsédé par l'informatique, quoiqu'on puisse élargir le terme à l'ensemble des fanatiques de disciplines scientifiques. Il sert aussi à qualifier un clown de carnaval aussi bien qu'une situation ou un être décalé, dégénéré. Ces différentes définitions collent à la narration proposée par Dunn.

L'histoire porte sur une fête foraine itinérante, I'Univers fabuleux des Binewski, propriété d'Al Binewski et de sa femme Crystal Lil (Lilian Hinchcliff). Les affaires périclitent et Al, de concert avec Lil, décide de se servir du corps de celle-ci pour créer de nouvelles attractions : «Il décida d'engendrer son propre spectacle de phénomènes. » (p. 16) Lil crée des monstres viables, des mutants qui deviendront d'intéressantes attractions foraines. Pendant ses grossesses, elle subit des radiations, boit de l'insecticide et des doses minimes d'arsenic, prend de la cocaïne, des amphétamines, sans craindre les mélanges. Il faut dire que Lil n'a pas froid aux yeux. Al en tomba amoureux lorsqu'elle accepta de proposer un spectacle au cirque au cours duquel elle tuait des poules en leur arrachant la tête. Avec ses dents.

Pendant leurs jeunes années, les enfants demandent souvent à leur père de raconter leur conception. Comme la bonne nourrice qu'on devine derrière les contes de la Mère I'Oye, Al Biniewski narre cette belle histoire qu'on qualifiera d'anti-pharmacopée et qui se termine toujours de semblable manière: «"L'idée me tomba dessus, comme ça, claire et 
complète, sans avoir à y penser trop longtemps." Il comprit qu'on pouvait concevoir les enfants comme ces roses. [...] Nous les enfants, nous souriions, l'embrassions, et il nous contemplait d'un air réjoui [...].» (p. 18) Nous sommes dans l'ordre du conte, dans I'univers du merveilleux. Il ne faut pas s'en étonner: «Le monstrueux est du merveilleux à rebours, mais c'est du merveilleux malgré tout. D'une part, il inquiète: la vie est moins sûre d'elle-même qu'on avait pu le penser. D'autre part, il valorise: puisque la vie est capable d'échecs, toutes ses réussites sont des échecs évités. » (Canguilhem, 1998, p. 173) Le monde du cirque se situe hors du quotidien, dans un univers onirique. Ou dans un univers dantesque, au vu des résultats obtenus par Al et Lil. Jusqu'au milieu du $\mathrm{XX}$ siècle, on croyait l'utérus une barrière infranchissable: le fœtus ne pouvait être perturbé par des substances provenant de l'extérieur. Les connaissances ont évolué. Al et Lil en prennent acte. Faut-il s'étonner qu'Al ait eu un intérêt pour les sciences médicales? Il a découvert la médecine sur le tas, devenant un modèle plutôt original de savant fou.

Quatre enfants meurent à la naissance, d'autres à un très jeune âge (dont une fille-lézard, à la grande déception du couple) et sont conservés dans des bocaux. Quatre survivent: Arturo a des nageoires au lieu de membres et ressemble à un phoque. Il donne d'époustouflants spectacles nautiques. Electra et Ephigenia, soeurs siamoises attachées par le bassin, donnent des concerts à quatre mains. Fortunato, le benjamin, semble tristement normal et ses parents veulent s'en débarrasser, jusqu'à ce qu'ils découvrent ses pouvoirs psychokinétiques phénoménaux. La narratrice, Olympia, se considère comme la ratée de la famille. Sans dons spéciaux, sa différence saute pourtant aux yeux puisqu'il $s^{\prime}$ agit d'une naine bossue, chauve et albinos. Elle possède une bonne voix et sert comme rabatteuse pour attirer le public. Des gens pareils attirent des êtres bizarres: Vern Bogner n'a plus de visage (il ne lui reste qu'un œil après une tentative ratée de suicide au pistolet) et devient un disciple fanatique d'Arturo; le docteur Phyllis, spécialiste des amputations et dont les règles éthiques n'ont rien à envier aux médecins nazis, a été expulsée de l'école de médecine malgré de très bons résultats parce qu'elle tentait une opération chirurgicale... sur son propre foie; le journaliste Norval Sanderson, obsédé par la troupe et surtout par Arturo, se fait lentement découper en morceaux, etc. Ces individus, aimantés par les membres de la famille, sans être des aberrations chromosomiques comme ces derniers, offrent néanmoins des corps en déliquescence ou hantés par la destruction.

Cet univers chaotique dans lequel on entre, ces phénomènes de foire par lesquels nous sommes happés, composent une narration qui mène au désastre. Profondément (et brillamment) grinçant, cynique, I'univers de Dunn n'a en tout cas rien de joyeux. Drôle parfois, à sa manière, il rappelle plus certaines toiles de James Ensor ou les Sabbat de Goya que le rire de Rabelais. La norme à partir de laquelle le carnavalesque se pense ne se trouve nulle part; I'informe, partout. Le spectaculaire désastre en marche ne manque pas d'effets pyrotechniques. Il permet de proposer une lecture politique du roman. 
Il existe une ambivalence certaine au cirque en soi, et à certains zoos. L'exhibition peut révéler une grande cruauté. À une certaine époque plus encore qu'aujourd'hui. Cette cruauté ne se dissocie pas de la définition du monstre. Il désignait encore, il y a peu, simplement l'Autre. «Monstre» vient du latin monstrare qui signifie avertir, signaler, et le verbe «démontrer» y trouve son origine étymologique. Je suggère qu'Un amour de monstres traduit de manière grotesque un phénomène que l'Occident a largement oublié, caché sous le tapis: I'exposition de ceux considérés sans arrière-pensée comme des monstres. Il avertissait d'un danger: I'invraisemblance de ce qu'on voyait, devant soi, n'avait rien d'humain et la dégénérescence ressemblait à ça.

Le roman de Dunn actualise un phénomène né aux États-Unis et qui touchera vite l'Europe. Le cirque Barnum, fondé au début des années 1840, en plein cœur de Manhattan, fait du freak show le clou de ses spectacles. D'abord nommé American Museum, il permet d'exhiber des femmes à barbe et des jumeaux siamois, des prodiges dépourvus de bras et de jambes et des hermaphrodites. On les présente dans un cadre ritualisé «qui se saisit de toute déviation de la norme, puis l'embellit et I'accentue pour produire un spectacle humain dont chaque trait somatique se trouve dès lors chargé de signification aux yeux du spectateur fasciné. » (Garland-Thomson, 2004, p. 42) L'exhibition de ces monstres, théâtralisée, mise à distance, permet au spectateur de sentir avec réconfort sa propre banalité. Cet exotisme dépasse rapidement le cadre de singularités physiques pour toucher un ensemble d'individus (appelonsles de manière neutre «visuellement différent») face à l'Européen qui représente l'étalon de la normalité. On oublie souvent que pendant près d'une centaine d'années se développent, parallèlement aux cirques, aux phénomènes de foire et aux zoos animaliers, des zoos humains mettant en scène des peuplades sauvages mises en scène - derrière des grilles pour ne pas effaroucher les spectateurs, nombreux. Car pendant des décennies, jusqu'à la fin des années 1920, il s'agit d'une culture de masse, aussi populaire que les expositions universelles. On va jusqu'à mettre des écriteaux enjoignant aux visiteurs de ne pas nourrir les sauvages: on s'occupe déjà de leur bien-être.

Les zoos humains répondent aux fantasmes et aux inquiétudes de I'Occident sur l'ailleurs et donnent une réalité au discours racial alors en construction. [...] [P]remier contact, ici, entre les Autres et Nous, [cet] autre [est] importé, exhibé, mesuré, montré, disséqué, spectularisé, scénographié, selon les attentes d'un Occident en quête de certitudes sur son rôle de «guide du monde», de «civilisation supérieure». (Bancel et al., 2004, p. 5)

Aux États-Unis, les zoos humains se développent davantage à travers les phénomènes de foire et attirent beaucoup les eugénistes.

On imagine la violence de ces exhibitions, de leur mise en forme: «La violence est partout. Tout autant dans le regard, dans le normal, dans le banal, dans le côté "bon enfant" de ces exhibitions, que dans les crimes les plus visibles.» (Bancel et al., 2004, p. 10) Car crimes - sadisme, torture, traitement inhumain en général - il y eut. Le raffinement, la 
théâtralisation de l'exhibition n'apporte pas que plaisir au spectateur. «Cela permet de se protéger contre la présence immédiate de l'autre monstrueux, tout en conservant un peu de l'émotion de ce qui aurait pu être la rencontre réelle. » (Ancet, 2006, p. 56) Cette mise à distance, rationnalisée à l'ère du positivisme scientifique (on parle de monstre, de déviant, de race inférieure, d'être qui ne trouve pas sa place dans la hiérarchie humaine), légitime l'indifférence face à la souffrance.

Un amour de monstres perpétue cette tradition, la renverse, dans un cadre contemporain et du point de vue du monstre - la narration enferme le lecteur dans le cirque ambulant. Ce qu'on connaît de l'extérieur vient d'individus qui se racontent parce qu'ils veulent adhérer au cirque et à ses valeurs. Les enfants détestent lorsque le père s'éloigne pour affaires. «La fête foraine était notre univers et celui de papa. Cela nous suffisait. Aucun d'entre nous n'avait jamais dormi dans un hôtel, mangé dans un restaurant, ou pris un avion. [...] Nous ne pouvions vivre que dans l'enceinte de la fête. » (p. 101) «L'Autre» désigne I'individu qui se croit normal. Non seulement les membres de la famille Binewski ne se considèrent pas comme des monstres (plutôt comme des êtres singuliers, sinon exceptionnels), mais le roman ne soulève jamais de questions éthiques liées au choix des parents. Il n'existe pas de point de vue extérieur pour les juger. Olympia l'affirme clairement: «Les gens de l'extérieur n'avaient aucune réalité pour moi.» (p. 233)

La violence, effrayante, vient de l'intérieur. Lil et Al ont créé des monstres grâce à des expériences biochimiques et pharmacologiques. Un des monstres va leur échapper, devenir un sommet de monstruosité morale, la vengeance du zoo humain, la réponse du freak show, miroir déformant d'une humanité qui se croit saine. Foucault a décrit comment, à partir du

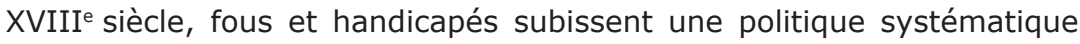
d'enfermement. Dans Un amour de monstres, les «enfermés» s'attaquent aux gardiens...

Arturo, I'aîné, en bon héritier, devient l'épicentre de la monstruosité. Il représente l'ambivalence propre au monstre qui fascine, attire et révulse à la fois. Cet effet s'exprime déjà au sein de sa famille. Electra et Ephigenia représente une figure de Janus: I'une déteste et craint Arturo, l'autre lui voue une immense admiration. L'ambiguïté de la position d'Olympia ne se dément pas: peur, attirance, jalousie, envie, dévotion? Elle tente de s'expliquer à ce sujet, sans succès : «[]]e ne baptiserais pas amour, le sentiment que j'éprouvais pour Arty. Disons plutôt, obsession. Mon obsession pour Arty était une maladie, non contagieuse, qui reste incompréhensible, même pour moi, malgré les années passées.» (p. 353) Quoiqu'il en soit, elle fait ses quatre volontés. Quant à Fortunato, le plus jeune, il aura peur de son frère, avant d'être à son service, puis finalement de le détruire, comme le cirque, ce qui l'entoure, et sa famille presque au complet, dans une grande finale apocalyptique.

Arturo, monstre postmoderne - j'entends par là qu'il naît grâce aux possibilités biotechnologiques d'un univers postindustriel où I'hybridité, la différence, le mélange, s'impose comme norme - possède aussi les 
attributs du monstre mythologique, métaphore du mal, et condense les traits du monstre imaginaire.

Envieux, jaloux, irascible, il ne supporte pas le succès des sœurs siamoises (qu'il torture), tente de tuer Fortunato et a sans doute assassiné sa sœur-lézard dont il craignait le succès potentiel. Peu à peu, la famille se trouve sous son joug et les attractions du cirque à la remorque du «spectacle» que propose Arturo: celui d'un chef de secte divinisé, assoiffé de pouvoir, qui manipule ses sectateurs de manière sadique, attire les foules pour les homogénéiser, à son image et à sa ressemblance. Ses apôtres les plus fanatiques suivent ses préceptes et acceptent, grâce aux bons soins du docteur Phyllis, de se faire découper en morceaux jusqu'à ce qu'il ne reste d'eux qu'une tête et un torse. Un état de dénuement complet, un corps sans mobilité, semblable à celui d'Arturo, mais plus démuni - ce dernier a besoin d'aide lorsqu'il se trouve sur le plancher des vaches, mais il possède des nageoires qui lui permettent de s'exhiber dans l'eau.

Le cirque devient lui-même un monstre, une incongruité hypertrophique qui échappe à son fondateur, Al Biniewski. L'Univers fabuleux des Binewski prend une expansion démesurée, avec des gardes en nombre grandissant qui protègent Arturo, et une horde de pèlerins qui suivent.

Les difformités spectaculaires d'Arturo font de lui un monstre marin. Or, le plus connu des monstres marins (peut-être de tous les monstres), auquel on compare Moby Dick par exemple, reste le Léviathan, figure mythique du livre de Job. Le Léviathan donne aussi son titre à un célèbre ouvrage de philosophie politique, celui de Thomas Hobbes. Le monstre biblique devient métaphore de l'état moderne tout-puissant, créé artificiellement par la volonté des individus qui se dépossèdent de leur liberté naturelle pour accéder aux avantages de l'existence civile, pour le bien commun. «En rendant raisonnable le monstre par l'artifice, en le transformant en un instrument efficace, une telle philosophie moderne de I'État peut-elle vraiment effacer la "monstruosité naturelle" de la multitude en révolte, des exclus du pouvoir politique?» (Guilhaumou, 2005, p. 184). Arturo joue de son charisme qui tient pour une part à son état monstrueux pour profiter d'une foule qui ne demande qu'à se laisser aller à la déraison.

L'image du Léviathan accentue la figure du monstre politique, mais le politique s'exprime aussi dans le nom de la secte: «Arturisme» ou «culte arturien». Nous sommes projetés dans l'univers légendaire du cycle du roi Arthur. Arturo et ses frères et sœurs sont des êtres de légendes: leur père les a bercés de ses romances sur le monde merveilleux de leur naissance. Monde de légendes, de mythe, mais aussi de pouvoir hiérarchique par le biais d'un monde monarchique revisité. Accréditant par ailleurs l'image propre aux familles royales dégénérées et décadentes, deux adjectifs qui collent aux Binewski, Arturo a un enfant avec Olympia. Relation contre-nature dont on ne peut le considérer responsable, puisque sa soeur subtilise son sperme grâce à l'aide de son frère Fortunato, appelé à remplacer le docteur Phyllis et spécialiste de la 
manipulation des corps. Les anciens monarques avaient des relations incestueuses, maintenant ils deviennent donneurs de sperme pour les membres de leur famille. Olympia demandera simplement l'aide de Fortunato: «Pourrais-tu faire bouger ce sperme - ces petites choses frétillantes - pourrais-tu les faire venir en moi, les placer dans mon œuf, pour que je puisse avoir un bébé comme Iphy?»(p. 333) Paul Valéry disait que «Le complément nécessaire d'un monstre c'est un cerveau d'enfant. » (cité dans Canguilhem, 1998, p. 17) Les membres de la famille, à commencer par Arturo, réagissent justement par des comportements infantiles, réactions impulsives, irrationnelles et immédiates. Le champion sera Fortunato, le clinamen, l'erreur, l'enfant à la normalité apparente. Dans une crise de haine à l'égard de son frère, il use de ses pouvoirs pour détruire le cirque et ceux qui y vivent, lui compris. Ne survivront qu'Olympia qui reste pour raconter cette histoire - comme Ishmael survit à l'attaque du Léviathan - ainsi que Lil, qui ne sait trop où elle en est. Et Miranda, I'enfant qui aura été éloignée de ce monde. L'Apocalypse aura été accomplie, au moment où Fortunato a une Révélation.

Avec la génétique, n'importe quoi peut arriver. Les enfants du couple Al et Lil furent conçus, avec l'aide de produits malsains, dans la perspective d'un eugénisme négatif (créer des dégénérés, des monstres, débilitant la «race»). Miranda, la fille issue de parents difformes, sera normale. Ou presque. Elle possède une petite queue au-dessus du derrière. Olympia l'a déposée dans un orphelinat et la regarde de loin faire son chemin. Parce qu'une femme riche veut lui payer une opération pour faire disparaître sa queue se produira la dernière tragédie de ce roman apocalyptique. Olympia s'introduit dans le monde de cette riche héritière pour la tuer - on n'a pas le droit de changer sa fille - , mais les deux femmes s'assassineront mutuellement dans l'ultime scène grotesque d'Un amour de monstres. Olympia lègue à sa fille des textes qui lui révèlent la vérité en détail, de l'argent et une grand-mère gâteuse.

À l'ère de la défense de la différence, Arturo impose sa dictature sans opposition. Pourquoi la différence serait-elle meilleure que la ressemblance, le faible davantage pur que le fort? Rappelons un élément fondamental de la nature humaine: le pouvoir corrompt peut-être, mais il rend d'abord bête et méchant. Le pouvoir s'oppose à la pensée socratique. Socrate dit : j'énonce pour te provoquer, t'obliger à réfléchir et à répondre. Le pouvoir dit: «Tais-toi». Vouer un culte signifie se taire et suivre. Les leaders charismatiques ne peuvent pas ne pas le savoir; voilà pourquoi ils deviennent si facilement monstrueux.

\section{BIBLIOGRAPHIE}

ANCET, Pierre (2006). Phénoménologie des corps monstrueux, Paris, Presses universitaires de France.

BANCEL, N., P. BLANCHARD, G. BOËTSCH, É. DEROO, R. GARLAND-THOMSON. «Introduction», dans BANCEL, N., P. BLANCHARD, G. BOËTSCH, É. DEROO, R.

GARLAND-THOMSON (dir.), Zoos humains: au temps des exhibitions humaines, Paris, La Découverte/poche, 2004, p. 5-18. 
CANGUILHEM, Georges (1998 [1965]). La connaissance de la vie, Paris, J. Vrin.

GARLAND-THOMSON, Rosemarie (2004). «Du prodige à l'erreur : les monstres de I'Antiquité à nos jours», dans N. BANCEL, P. BLANCHARD, G. BOËTSCH, É. DEROO, et S. LEMAIRE (dir.), Zoos humains: au temps des exhibitions humaines, Paris, La Découverte/poche.

GUILHAUMOU, Jacques (2005). «Les monstres dans l'ordre social: généalogies du "monstre en politique" de Machiavel à Sieyès», dans R. Bertrand et A. Carol (dir.), Le monstre humain: imaginaire et société, Aix-en-Provence, Publications de I'Université de Provence, p. 179-190.

\section{Christian Chelebourg \\ Les Écofictions. Mythologies de la fin du monde}

Bruxelles, Les impressions nouvelles, coll. «Réflexions faites», 2012, 254 p.

\section{Patrick Bergeron}

Professeur agrégé, Département d'études françaises, Université du Nouveau-Brunswick

«Au calendrier de I'Apocalypse, écrit Christian Chelebourg, une date chasse l'autre. » Celle du 21 décembre 2012, qui correspond à la fin du $13^{\text {e }}$ baktun (cycle long) dans le calendrier maya, s'ajoute ainsi à une liste déjà longue de dates faussement fatidiques. On sait maintenant que I'humanité a encore du temps devant elle. Toutefois, l'idée selon laquelle la Terre et I'Homo sapiens sont en péril ne cesse depuis quelques années de refluer dans l'imaginaire collectif. On n'a qu'à se rappeler les récents films de Roland Emmerich et de Lars von Trier, 2012 (2009) et Melancholia (2011). Si, techniquement parlant, la fin du monde n'a pas encore eu lieu, elle est constamment reconduite dans le domaine de la fiction (surtout dans la littérature et au cinéma). C'est de ce phénomène de société que rend compte Christian Chelebourg en prenant appui sur une vaste sélection de romans, de films, de bandes dessinées, de documentaires, d'essais et de publicités. Ceux-ci ont en commun d'exploiter les nouvelles peurs qui secouent jour après jour les sociétés industrielles: pollution, réchauffement climatique, effet de serre, perforation de la couche d'ozone, désastres naturels, épidémies, parmi d'autres fléaux contemporains. Tantôt, d'anciens mythes sont réactivés, tantôt de nouveaux sont créés. Les signes de l'apocalypse sont donc partout. Un demi-siècle après le grand classique de Roland Barthes, Chelebourg propose à son tour une étude de nos «mythologies». C'est d'ailleurs le sous-titre qu'il a donné à son ouvrage: Mythologies de la fin du monde.

Professeur à I'Université de Lorraine où il dirige l'équipe d'accueil «Littérature, Imaginaire, Sociétés» (anciennement Centre d'études littéraires Jean Mourot), Christian Chelebourg est spécialiste de littérature française du XIX siècle (Mérimée, Hugo, Verne), d'imaginaire et de fiction pour la jeunesse, sujet de son plus récent livre (Les fictions de jeunesse, PUF, 2013). 